\title{
Encuesta sobre el uso de Medicina alternativa y complementaria en pacientes mexicanos con cáncer atendidos en un centro de referencia oncológico
}

\author{
Mariana Navarro-Hernández¹, Nancy Reynoso-Noverón² y Amanda De la Piedra-Gómez ${ }^{1 *}$ \\ ${ }^{1}$ Centro de Apoyo para la Atención Integral; ${ }^{2}$ Dirección de Investigación. Instituto Nacional de Cancerología, Ciudad de México, México
}

\begin{abstract}
Resumen
Introducción: El uso de Medicina alternativa y complementaria (MAC) se ha incrementado en pacientes con cáncer, pero los datos sobre su uso son escasos. Objetivo: Describir la frecuencia de uso de MAC en pacientes oncológicos atendidos en el Instituto Nacional de Cancerología. Material y métodos: Se aplicó una encuesta estructurada y validada sobre datos sociodemográficos y uso de MAC. Resultados: Se incluyeron 492 pacientes con cáncer de mama, cérvix y pulmón principalmente, el $76.6 \%$ eran mujeres. El ejercicio, suplementos alimenticios y herbolaria se reportó en un $80 \%$, fue más frecuente en mujeres de 51-66 años y con más de cuatro años de diagnóstico. La escolaridad y el tipo de neoplasia se asociaron con el uso de estas terapias. Conclusiones: El uso de MAC es frecuente en pacientes oncológicos. Es necesario identificarlas a fin de orientar sobre su uso y considerarlas en el tratamiento integral del cáncer.
\end{abstract}

Palabras clave: Medicina alternativa. Terapias complementarias. Cáncer.

\section{Survey on the use of alternative and complementary medicine in mexican patients with cancer in an oncology reference center}

\section{Abstract}

Objective: To describe the frequency of use of alternative and complementary medicine (CAM) and its relationship with sociodemographic, economic and clinical variables in oncology patients treated at the National Cancer Institute. Material and methods: A structured and validated survey for a certain sociodemographic population was applied to measure the use of CAM. Results: We included 492 patients with breast, cervix and lung cancer mainly, $76.6 \%$ were women. The main diagnosis was breast cancer (37.4\%). Exercise, dietary supplements and herbal products were reported in $80 \%$; higher frequency in women aged 51-66 years and with more than four years diagnosis. Schooling and the type of neoplasia were associated with the use of these therapies. Conclusions: The use of CAM is common in oncologist patients. It is necessary to identify them in order to guide their use and consider them in the integral management of cancer.

Key words: Cancer. Integrative Oncology. alternative medicine. complementary therapies.

\section{Correspondencia:}

*Amanda de la Piedra-Gómez E-mail: amandadpg@ hotmail.com
Disponible en internet: 20-03-2019

Gac Mex Oncol. 2018;17:237-244 www.gamo-smeo.com 1665-9201/@ 2018 Sociedad Mexicana de Oncología. Publicado por Permanyer México SA de CV. Este es un artículo Open Access bajo la licencia CC BY-NC-ND (http://creativecommons.org/licenses/by-nc-nd/4.0/). 


\section{Introducción}

El cáncer es una de las principales causas de enfermedad y muerte en el mundo. En 2012, hubo 14 millones de casos nuevos y 8.2 millones de muertes relacionadas con esta patología ${ }^{1}$. Se estima que el número de casos nuevos se incrementará un 70\% aproximadamente en los próximos 20 años ${ }^{2}$. En México se detectan cerca de 150 mil casos de cáncer al año y es la segunda causa de muerte en la población general ${ }^{3}$. Según Gómez-Dantés, et al., en México en 2013 se registraron 84,172 defunciones por cáncer, de las cuales 41,281 (\%) corresponden al sexo masculino y 42,891 (\%) al femenino. Las principales causas de muerte en hombres fueron cáncer de próstata y de pulmón (6,296 y 4,276 defunciones, respectivamente). Para el caso de las mujeres fueron cáncer de mama (5,997 defunciones), del cuello del útero $(4,076)$ y del hígado y de las vías biliares intrahepáticas $(3,124)^{4}$. Este grupo de enfermedades crónicas se asocia con un alto costo físico, social y económico ${ }^{5}$. En la actualidad existen muchos tipos de tratamientos convencionales para el cáncer y su indicación depende del tipo de padecimiento oncológico y la etapa clínica en que este se encuentre. Sin embargo, en muchos casos los resultados no son del todo positivos para el paciente, producen efectos secundarios serios y no se alcanza el control de la enfermedad 6 .

Por esta razón, muchos pacientes optan por utilizar de manera combinada la Medicina alternativa y complementaria (MAC), término que se utiliza para designar aquellos productos y prácticas médicas que se considera que no pertenecen a la atención médica convencional. La medicina complementaria se usa junto con tratamientos médicos convencionales y la medicina alternativa se usa en lugar del tratamiento habitual ${ }^{7}$.

Según la Organización Mundial de la Salud, los términos de Medicina complementaria/alternativa se utilizan de manera indistinta junto con la medicina tradicional en algunos países y hacen referencia a un conjunto amplio de prácticas de atención de salud que no forman parte de la propia tradición del país y no están integradas en el sistema sanitario y de salud de la seguridad social ${ }^{8}$. La MAC abarca una amplia variedad de terapias y prácticas y se ha venido utilizando desde hace miles de años ${ }^{9}$. El resurgimiento de su utilización en muchos países desarrollados y en desarrollo se ha presentado a partir de los años noventa del siglo XX y han sido reconocidas como componentes, usadas para el cuidado y apoyo de pacientes con enfermedades cronicodegenerativas $^{10-12}$. En este sentido, la oncología integrativa enfatiza la toma de conciencia y la sensibilidad respecto a las necesidades mentales, emocionales y espirituales del paciente ${ }^{11}$. En México, por razones sociales y culturales, el uso de medicina tradicional, herbolaria y remedios caseros de origen indefinido es sumamente frecuente ${ }^{12,13}$.

El objetivo de este estudio fue analizar el tipo y frecuencia de consumo de estos tratamientos en pacientes con cáncer que acuden para recibir atención médica al Instituto Nacional de Cancerología (INCan), así como su posible asociación con su perfil sociodemográfico y clínico.

\section{Material y métodos}

Para recopilar los datos sobre el uso de MAC se realizó un estudio transversal con aplicación de un cuestionario elaborado con base en las guías de la Society for Integrative Oncology, que tenía en consideración diversas prácticas de tratamientos alternativos y complementarios ${ }^{14}$. Este cuestionario se aplicó de enero a marzo de 2011 a 500 pacientes atendidos en el INCan y que se encontraban en las salas de espera. Todos ellos con capacidad de entendimiento del lenguaje del cuestionario, en condiciones para completarlo por sí mismos y que voluntariamente aceptaron participar en este estudio.

Para el análisis de datos se calcularon medidas de tendencia central y dispersión. Las diferencias entre sexo, variables diagnósticas, socioeconómicas, demográficas y el uso de MAC fueron evaluadas mediante la prueba de Chi cuadrada $\left(\chi^{2}\right)$ o la prueba exacta de Fisher. Las variables que pudieran influir en el uso de MAC se incluyeron en un modelo regresión logística múltiple; dicho modelo se ajustó por sexo, edad, tiempo desde el diagnóstico, nivel educativo y diagnóstico, utilizando el software Stata/SE ${ }^{\circledR}$ versión 12.

\section{Resultados}

\section{Características sociodemográficas y socioeconómicas}

Participaron en el estudio 500 pacientes, 398 mujeres $(76.6 \%)$, con promedio de edad de $51.36( \pm 12.85)$ años, y 94 hombres (18.8\%), con una media de edad de $53( \pm 16.34)$ años; ocho sujetos $(1.6 \%)$ no especificaron el sexo, por lo que fueron excluidos del estudio. Los resultados que aquí se presentan corresponden a 492 participantes. 
Tabla 1. Características sociodemográficas, económicas y clínicas de la población de estudio

\begin{tabular}{|c|c|c|c|c|c|}
\hline & \multicolumn{2}{|c|}{ Hombres } & \multicolumn{2}{|c|}{ Mujeres } & \multirow[t]{2}{*}{$p<z$} \\
\hline & $\mathrm{N}^{0}$ de personas & $\%$ & $\mathrm{~N}^{0}$ de personas & $\%$ & \\
\hline Sexo & 94 & 19.11 & 398 & 80.89 & \\
\hline $\begin{array}{l}\text { Nivel educativo } \\
\text { Analfabeto } \\
\text { Primaria } \\
\text { Secundaria } \\
\text { Preparatoria } \\
\text { Licenciatura } \\
\text { Posgrado } \\
\text { No especificado }\end{array}$ & $\begin{array}{c}8 \\
19 \\
28 \\
18 \\
11 \\
5 \\
5\end{array}$ & $\begin{array}{c}8.51 \\
20.21 \\
29.79 \\
19.15 \\
11.70 \\
5.32 \\
5.32\end{array}$ & $\begin{array}{c}22 \\
138 \\
107 \\
57 \\
57 \\
6 \\
11\end{array}$ & $\begin{array}{c}5.53 \\
34.67 \\
26.88 \\
14.32 \\
14.32 \\
1.51 \\
2.76\end{array}$ & 0.026 \\
\hline $\begin{array}{l}\text { Derechohabiencia } \\
\text { Seguro popular } \\
\text { No seguro popular } \\
\text { No especificado }\end{array}$ & $\begin{array}{c}8 \\
72 \\
14\end{array}$ & $\begin{array}{c}8.5 \\
76.6 \\
14.9\end{array}$ & $\begin{array}{c}166 \\
206 \\
26\end{array}$ & $\begin{array}{c}41.71 \\
51.76 \\
6.53\end{array}$ & 0.000 \\
\hline $\begin{array}{l}\text { Tiempo transcurrido desde el diagnóstico } \\
\text { menor a } 1 \text { año } \\
1 \text { año } \\
2 \text { años } \\
3 \text { años } \\
4 \text { años } \\
\text { mayor a } 5 \text { años } \\
\text { No especificado }\end{array}$ & $\begin{array}{c}32 \\
24 \\
14 \\
10 \\
1 \\
13 \\
0\end{array}$ & $\begin{array}{c}34.0 \\
25.5 \\
14.9 \\
10.6 \\
1.1 \\
13.8 \\
0.0\end{array}$ & $\begin{array}{l}127 \\
140 \\
33 \\
22 \\
16 \\
47 \\
13\end{array}$ & $\begin{array}{c}31.91 \\
35.18 \\
8.29 \\
5.53 \\
4.02 \\
11.81 \\
3.27\end{array}$ & 0.029 \\
\hline $\begin{array}{l}\text { Edad por categorías (años) } \\
\text { menores de } 34 \\
35 \text { a } 50 \\
51 \text { a } 66 \\
\text { mayores de } 67\end{array}$ & $\begin{array}{l}13 \\
27 \\
31 \\
23\end{array}$ & $\begin{array}{l}13.83 \\
28.72 \\
32.98 \\
24.47\end{array}$ & $\begin{array}{c}35 \\
156 \\
149 \\
58\end{array}$ & $\begin{array}{c}8.79 \\
39.2 \\
37.44 \\
14.57\end{array}$ & 0.027 \\
\hline $\begin{array}{l}\text { Estado de procedencia } \\
\text { Ciudad de México } \\
\text { Estado de México } \\
\text { Hidalgo } \\
\text { Morelos } \\
\text { Tlaxcala } \\
\text { Otros }\end{array}$ & $\begin{array}{c}26 \\
20 \\
9 \\
9 \\
4 \\
26\end{array}$ & $\begin{array}{c}27.66 \\
21.28 \\
9.57 \\
9.57 \\
4.26 \\
27.66\end{array}$ & $\begin{array}{l}139 \\
84 \\
35 \\
28 \\
16 \\
96\end{array}$ & $\begin{array}{c}34.92 \\
21.11 \\
8.79 \\
7.04 \\
4.02 \\
24.12\end{array}$ & 0.764 \\
\hline $\begin{array}{l}\text { Tipo de cáncer } \\
\text { Mama } \\
\text { Linfoma } \\
\text { Ginecológico } \\
\text { Esófago } \\
\text { Cervicouterino } \\
\text { Estómago } \\
\text { Sarcoma } \\
\text { Cabeza y cuello } \\
\text { Pulmón } \\
\text { Ovario } \\
\text { Tiroides } \\
\text { Otros } \\
\text { No especificado }\end{array}$ & $\begin{array}{c}0 \\
9 \\
0 \\
4 \\
0 \\
4 \\
6 \\
6 \\
14 \\
0 \\
3 \\
42 \\
6\end{array}$ & $\begin{array}{c}0.00 \\
9.57 \\
0.00 \\
4.26 \\
0.00 \\
4.26 \\
6.38 \\
6.38 \\
14.89 \\
0.00 \\
3.19 \\
44.68 \\
6.38\end{array}$ & $\begin{array}{c}184 \\
17 \\
12 \\
2 \\
40 \\
7 \\
9 \\
10 \\
15 \\
19 \\
11 \\
32 \\
40\end{array}$ & $\begin{array}{c}46.23 \\
4.27 \\
3.02 \\
0.50 \\
10.05 \\
1.76 \\
2.26 \\
2.51 \\
3.77 \\
4.77 \\
2.76 \\
8.04 \\
10.05\end{array}$ & \\
\hline
\end{tabular}

El $59 \%$ de los pacientes tenían principalmente un nivel educativo básico (primaria o secundaria), el 16\% había cursado la educación superior o posgrado, un 15\% mencionó haber estudiado educación media superior, un $6 \%$ fueron personas sin educación formal y el $3 \%$ no especificaron su escolaridad (Tabla 1). Por derecho-habiencia solo se consideró el tener o no seguro popular; el $35 \%$ de los participantes estaban afiliados a dicho sistema de protección. El $42 \%$ de las mujeres se encontraban adheridas, en comparación con solo el $9 \%$ de los hombres.

Respecto al tiempo transcurrido desde el diagnóstico, en el $66 \%$ de los casos era de un año o menos y en el $12 \%$ habían pasado más de cinco años; en ambos sexos los mayores porcentajes se observaron en 


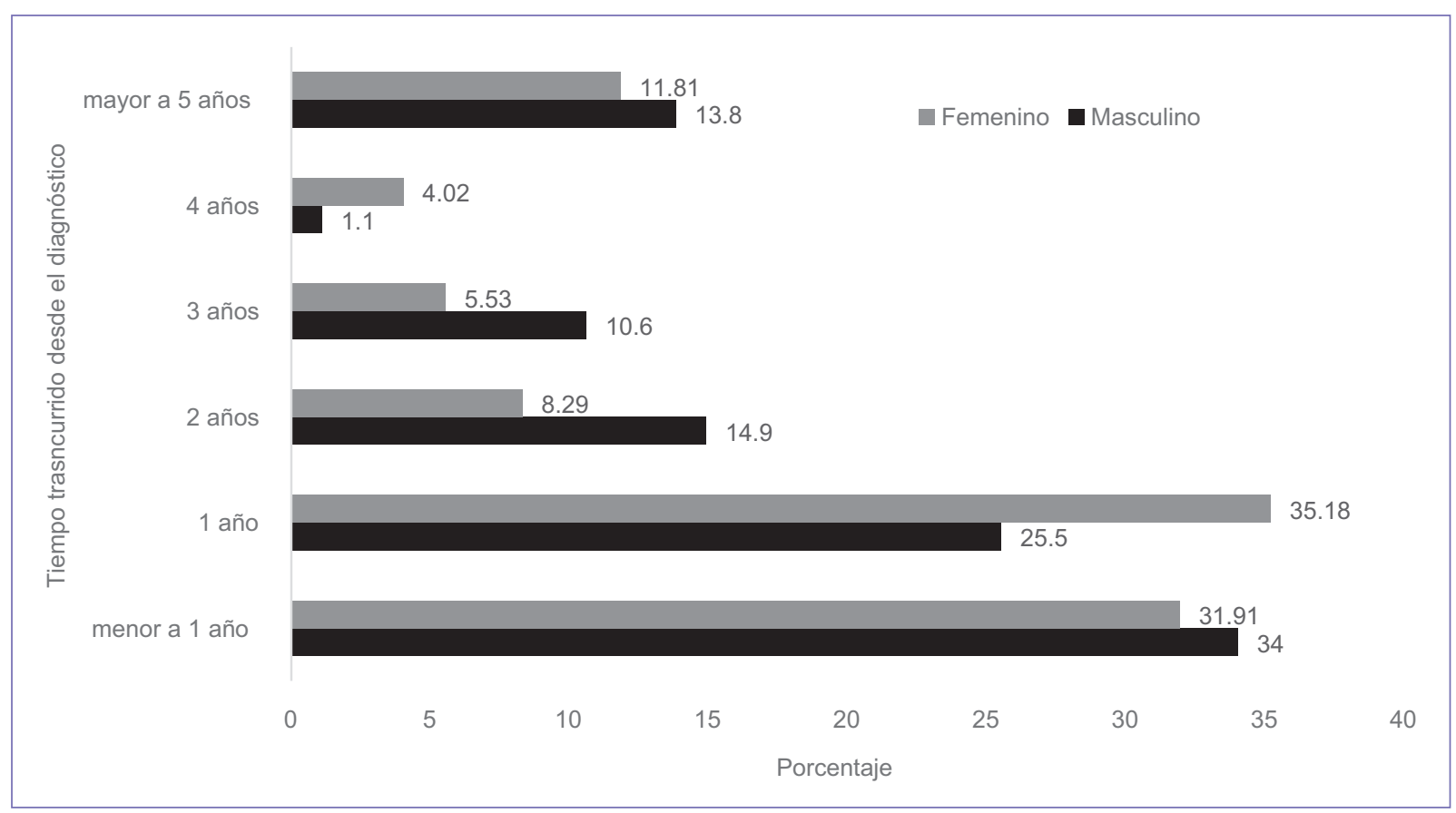

Figura 1. Porcentaje de pacientes que utilizan Medicina alternativa y complementaria (MAC) por tiempo transcurrido desde el diagnóstico por sexo.

las categorías de menos de un año o un año desde el diagnóstico (Fig. 1).

La mayoría de los pacientes provenían de la Ciudad de México (34\%) o del Estado de México (21\%).

La exploración del comportamiento de las variables según el sexo identificó diferencias estadísticamente significativas en nivel educativo ( $p=0.026$ ), derecho-habiencia $(p=0.000)$, tiempo transcurrido desde el diagnóstico $(p=0.029)$ y edad (establecida en cuatro categorías para el análisis) ( $p=0.027)$, pero no en relación con el lugar de procedencia.

Los principales tipos de cáncer reportados de manera general fueron de mama (37\%), cervicouterino (8\%) y de pulmón (5\%). En las mujeres, el cáncer de mama fue el más frecuente (46\%) y en los hombres el cáncer de pulmón (15\%).

Al indagar el uso de MAC, el 80.3\% (395) mencionó haberla utilizado, de estos el $82.28 \%$ fueron mujeres. Sin embargo, no se observaron diferencias significativas entre el sexo y el uso de MAC $(p=0.115)$.

La exploración por grupo de edad indicó que no había asociación entre esta y el uso de MAC. Sin embargo, fue más frecuente en el grupo de 51-66 años (83) y cabe señalar que en menores de 34 años el uso de MAC superó el $80 \%$ de uso.

Por tipo de cáncer, las mujeres que padecen cáncer de mama, cervicouterino, de ovario, ginecológico y sarcoma son las que en mayor medida han utilizado MAC. En todos los pacientes con cáncer de esófago se utilizó MAC; sin embargo, solo había seis pacientes con esta patología.

Según derecho-habiencia, los participantes con seguro popular son los que principalmente utilizan alguna MAC (87\%), presentándose diferencias significativas $(p=0.000)$ con respecto a los pacientes con otro tipo de derecho-habiencia. El análisis por nivel educativo mostró diferencias estadísticamente significativas $(p=0.000)$; los mayores porcentajes de uso de MAC se observan en los niveles educativos más altos: preparatoria, posgrado y licenciatura (95, 91 y $85 \%$ respectivamente).

Por tiempo transcurrido desde el diagnóstico, los que llevan cuatro años con el padecimiento son los pacientes que en mayor medida utilizan MAC (88\%), los que hacen menor uso de medicinas alternativas son los que fueron diagnosticados hace menos de un año (76) (Fig. 1).

Por tipo de MAC, el ejercicio y el consumo de suplementos alimenticios son los métodos más utilizados en la población de estudio (39 y 37\% respectivamente); la herbolaria y la medicina tradicional mexicana también son métodos alternativos usados por gran parte de los pacientes (34 y 20\% respectivamente) (Fig. 2).

Según el motivo de uso, el 31\% mencionó que fue para sentirse mejor y el $15 \%$ utiliza las MAC para 


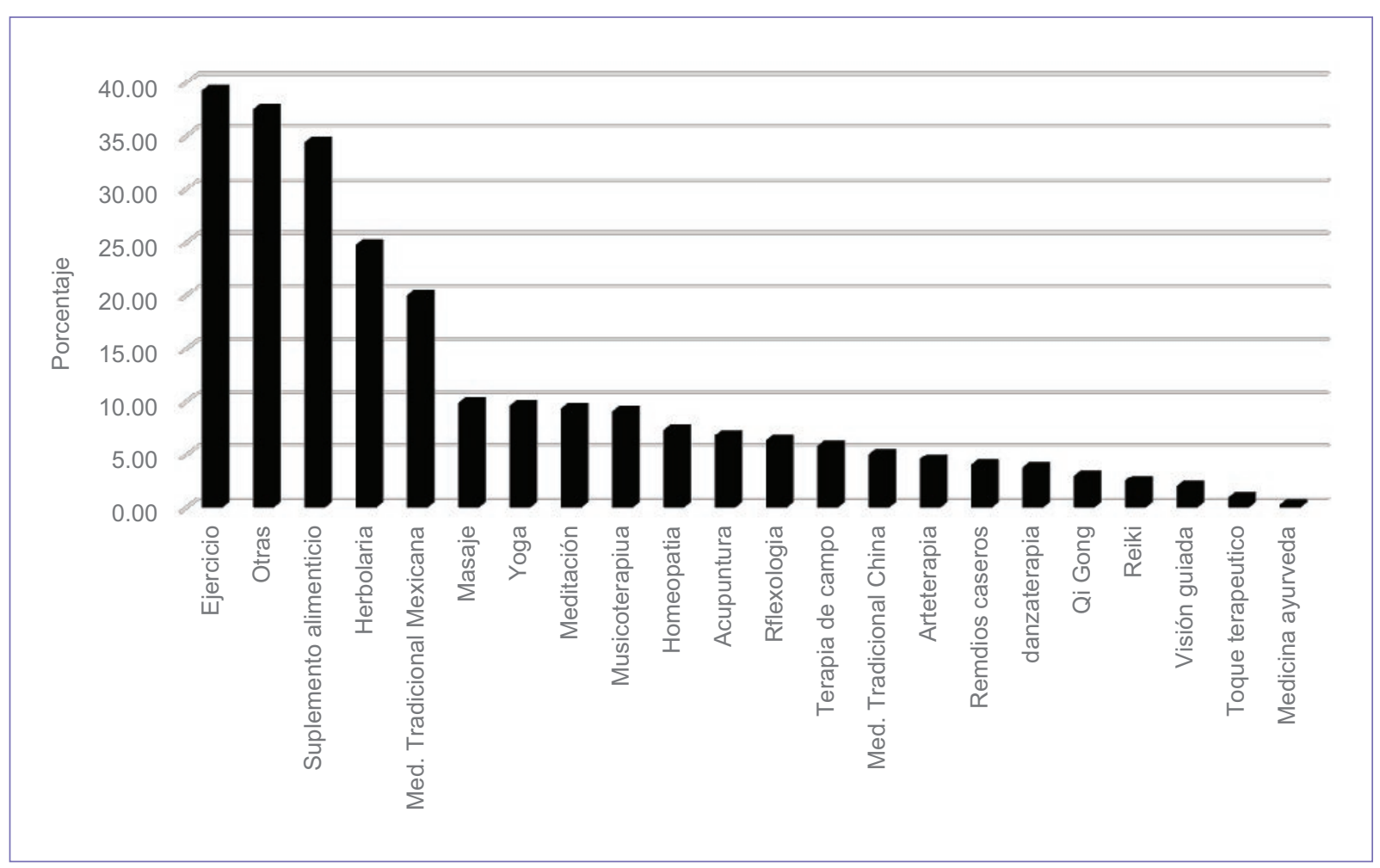

Figura 2. Frecuencia de uso de terapias de Medicina alternativa y complementaria (MAC). Cada uno de los 395 pacientes que utiliza MAC mencionó todos los tipos de MAC que utiliza, desde 1 hasta 21 ( $\mathrm{n}=395$ ).

combatir el cáncer; empero, el $9 \%$ de los encuestados no dio razón por la cual se mantenga empleando la medicina alternativa (Tabla 2).

De los pacientes que especificaron la frecuencia en el uso de las MAC, un $53 \%$ las utiliza más de tres veces por semana, un $12 \%$ las usa una vez por semana, el uso de dos veces por semana y ocasionalmente presenta porcentajes similares (11\%), un $8 \%$ de los pacientes la usa tres veces a la semana y solo el $4 \%$ una sola vez al mes.

Para que los pacientes comenzaran a utilizar alguna $M A C$, esta fue recomendada principalmente por un médico (32\%) o un amigo (19\%), el 17\% comenzó con las medicinas alternativas por iniciativa propia, el $24 \%$ por recomendación familiar, el $4 \%$ al recibir orientación en el Centro de Apoyo para la Atención Integral y un porcentaje equivalente por recomendación de alguna otra persona.

En la regresión logística se identificó que el nivel educativo está asociado de forma significativa al uso de MAC, el haber cursado la secundaria, preparatoria, licenciatura o posgrado incrementa la posibilidad de utilizar algún tratamiento no convencional, observándose la mayor asociación cuando los pacientes oncológicos han cursado el nivel medio superior (odds ratio [OR]: 27.11; intervao de confianza [IC]: 95\%,1.65158.01). El tipo de cáncer que se padece mostró asociaciones negativas estadísticamente significativas; en específico, el tener el diagnóstico de linfoma (OR: 0.31; IC: 0.10-0.94) o cáncer de pulmón (OR: 0.13; IC: 0.050.36 ) se relaciona con utilizar en menor medida la medicina alternativa (Tabla 3).

\section{Discusión}

La MAC se aprecia por diversos motivos y se utiliza de manera amplia en la mayoría de los países del mundo y este consumo está aumentando rápidamente. Esta práctica es en la actualidad para millones de personas la principal fuente de atención sanitaria y a veces la única. Adicionalmente, es la más próxima a los hogares, es accesible, asequible y culturalmente aceptada; muchas de las personas que la utilizan confían plenamente en su eficacia y seguridad ${ }^{15}$.

La prevalencia del uso de MAC en pacientes con cáncer es frecuentemente alta, se estima entre el 30 y el $90 \%{ }^{16,17}$. Publicaciones con pacientes oncológicos de países industrializados la reportan entre el 25 y el 
Tabla 2. Razones de uso de Medicina alternativa y complementaria

\begin{tabular}{|l|c|c|}
\hline Razones de uso & $\mathbf{N}^{0}$ de personas & $\%$ \\
\hline Sentirse mejor & 123 & 31.14 \\
\hline Combatir el cáncer & 59 & 14.94 \\
\hline Sintomatología & 35 & 8.86 \\
\hline Sistema inmunológico & 33 & 8.35 \\
\hline Apetito-anemia & 20 & 5.06 \\
\hline Efectos secundarios de la OT & 19 & 4.81 \\
\hline Ejercitarse & 14 & 3.54 \\
\hline Ansiedad y estrés & 13 & 3.29 \\
\hline Comorbilidades & 10 & 2.53 \\
\hline Otros & 32 & 8.10 \\
\hline No especificado & 37 & 9.37 \\
\hline Total & 395 & 100 \\
\hline
\end{tabular}

QT: quimioterapia.
Tabla 3. Variables asociadas con el uso de Medicina alternativa y complementaria

\begin{tabular}{|c|c|c|c|}
\hline Variable & Odds ratio & (IC 95\%) & $\mathbf{p}<\mathbf{z}$ \\
\hline Sexo & 0.84 & $(0.41-1.74)$ & 0.645 \\
\hline Edad & 1.02 & $(0.99-1.04)$ & 0.114 \\
\hline \multicolumn{4}{|c|}{$\begin{array}{l}\text { Tiempo transcurrido } \\
\text { desde el diagnóstico }\end{array}$} \\
\hline 1 a 2 años & 1.59 & $(0.87-2.92)$ & 0.136 \\
\hline 3 a 4 años & 1.29 & $(0.48-3.43)$ & 0.613 \\
\hline 5 años o más & 1.14 & $(0.47-2.75)$ & 0.772 \\
\hline \multicolumn{4}{|l|}{ Nivel educativo } \\
\hline Primaria & 1.28 & $(0.48-3.43)$ & 0.620 \\
\hline Secundaria & 3.66 & (1.25-10.68) & 0.018 \\
\hline Preparatoria & 27.11 & $(4.65-158.01)$ & 0.000 \\
\hline $\begin{array}{l}\text { Licenciatura y } \\
\text { posgrado }\end{array}$ & 3.42 & $(1.10-10.68)$ & 0.034 \\
\hline \multicolumn{4}{|l|}{ Tipo de cáncer } \\
\hline Linfoma & 0.31 & $(0.10-0.94)$ & 0.039 \\
\hline Cervicouterino & 1.02 & $(0.34-3.06)$ & 0.978 \\
\hline Sarcoma & 0.24 & $(0.40-1.40)$ & 0.112 \\
\hline Cabeza y cuello & 0.52 & $(0.12-2.22)$ & 0.376 \\
\hline Pulmón & 0.13 & $(0.05-0.36)$ & 0.000 \\
\hline Ovario & 0.55 & $(0.16-1.90)$ & 0.343 \\
\hline Otros & 0.33 & $(0.16-0.69)$ & 0.003 \\
\hline
\end{tabular}

IC: intervalo de confianza.

de menores de 34 y de 55 a ô ẫóos y con cáncer de mama, aunque las diferencias no fueron estadísticamente significativas.

En cuanto al nivel educativo, en la literatura se reporta que los pacientes con un nivel de escolaridad mayor tienden a usar más la MAC ${ }^{13,18,28}$. En esta muestra de pacientes oncológicos del INCan se encontró una asociación estadísticamente significativa con el consumo de MAC y un nivel de escolaridad superior a primaria.

En relación a la derecho-habiencia determinada únicamente por su afiliación o no al seguro popular, se halló que la mayoría de mujeres con enfermedad oncológica están afiliadas, lo cual es consistente con la inclusión en el Fondo para la Protección contra Gastos Catastróficos (FPGC) en 2004 y 2007 de los cánceres cervicouterino y de mama, sin embargo la proporción en hombres es menor, lo cual se debe a que el año en que se realizó el presente estudio fue el mismo en que se incluyó al cáncer de próstata en el FPGC (2011). El tiempo de diagnóstico se ha reportado en algunas investigaciones como importante en la decisión de usar o no MAC (significancia estadística) ${ }^{13,17}$, sin embargo en este estudio no se encontró asociación.

Al contrario de lo identificado en relación al tipo de cáncer, donde el padecer linfoma es un factor negativo, otros estudios han identificado que pacientes con linfoma son los que más utilizan $\mathrm{MAC}^{29}$, pero los datos 
son concordantes con el diagnóstico de cáncer de pulmón, dado que relativamente pocos pacientes con este diagnóstico utilizan alguna MAC ${ }^{21,30}$.

El $32.1 \%$ de las personas que usan MAC lo hacen por recomendación médica, proporción cercana a lo encontrado en literatura $(<30 \%)^{17}$. En este trabajo el personal de salud recomendó 7 de las 21 terapias usadas por los pacientes del INCan; las restantes las utilizaron por sugerencia de familiares, amigos y vecinos o iniciativa propia, entre otros, igual que lo informado en un estudio de $2015^{31}$.

En relación con las razones de uso, unas de las más frecuentes en esta población fueron fortalecer el sistema inmunológico y tratar los efectos secundarios de la quimioterapia, las cuales también fueron documentadas en otro estudio efectuado con los padres o cuidadores de niños con diferentes tipos de tumores malignos ${ }^{16}$.

Un factor no considerado en este estudio fue la inversión realizada por pacientes al uso de MAC. En la literatura, según reportes en EE.UU., la cantidad de dinero invertido en CAM ha ido en aumento en los últimos años; de acuerdo con los resultados de la encuesta en 2017, los estadounidenses gastaron $\$ 33.9$ mil millones en terapias CAM ese año. Se dividen los gastos en productos naturales, clases de instrucción y materiales, suplementos dietéticos y el resto de las visitas a practicantes de CAM como acupunturistas, masajistas o quiroprácticos ${ }^{32}$.

Se requiere ampliar la información del uso de las terapias complementarias, haciendo un análisis del costo-efectividad y el costo que representa para los pacientes el uso de CAM en México.

Es importante mencionar que la medicina no convencional es un componente importante y con frecuencia subestimado de los servicios de salud. Se ha utilizado de manera milenaria por muchas culturas de diferentes orígenes para mantener la salud, y prevenir y tratar las enfermedades, en particular las crónicas ${ }^{6}$.

En la actualidad, la mayoría de los centros oncológicos, incluido el INCan, han establecido programas de medicina integrativa para estudiar y combinar terapias útiles complementarias con el cuidado oncológico convencional y también fomentar entre la comunidad de pacientes el cuidado en el uso de terapias que no posean datos de seguridad y eficacia que puedan ser potencialmente perjudiciales. Su objetivo es complementar el tratamiento, la intervención y atención médica, tomando en consideración al individuo como un ser psicosocial. Esto implica que el personal médico debe emplear los mejores métodos de un amplio rango para sanar, tomando decisiones basadas en la evidencia.

Actualmente, uno de los grandes retos que afrontan las instituciones de salud y el público, así como las estancias gubernamentales en temas de normatividad, se relaciona con la seguridad y eficacia, la calidad, disponibilidad, preservación y reglamentación de la MAC. Es necesario que el sector salud en su conjunto identifique y conozca las diversas terapias alternativas que utilizan este tipo de pacientes, sus potenciales beneficios y riesgos, para así dar una mejor orientación sobre su uso y establecer terapias orientadas al beneficio del paciente oncológico aprovechando toda la gama de tratamientos existentes.

\section{Bibliografía}

1. Stewart BW, Wild CP, editores. World Cancer Report 2014 [Internet]. Lyon: International Agency for Research on Cancer [fecha de publicación: 2014]. Disponible en: https://www.drugsandalcohol.ie/28525/1/ World\%20Cancer\%20Report.pdf.

2. Cáncer. Datos y cifras [Internet]. Ginebra: Organización Mundial de la Salud [última actualización: 12 de septiembre de 2018]. Disponible en: http://www.who.int/mediacentre/factsheets/fs297/es.

3. Estilos de vida más saludables, para prevenir el cáncer [Internet]. Ciudad de México: Secretaría de Salud, Publicaciones México Sano [fecha de publicación: 2015]. Disponible en: http://www.gob.mx/salud/documentos/ publicaciones-mexico-sano-2015.

4. Gómez-Dantés H, Lamadrid-Figueroa H, Cahuana-Hurtado L, Silverman-Retana O, Montero P, González-Robledo MC, et al. The burden of cancer in Mexico 1990-2013. Rev Salud Publ Mex. 2016;58:118-31.

5. Valencia SC. Depresión en personas diagnosticadas con cáncer. Diversitas. 2006;2(2):241-57.

6. Managing Cancer-related Side Effects [Internet]. American Society of Clinical Oncology, Cancer.org [fecha de consulta: 12 de octubre de 2016]. Disponible en: http://www.cancer.org/treatment/treatmentsandsideeffects/physicalsideeffects/physical-side-effects-landing.

7. Complementary and Alternative Medicine [Internet]. Bethesda: National Cancer Institute [fecha última actualización: 10 de abril de 2015]. Disponible en: http://www.cancer.gov/about-cancer/treatment/cam

8. Medicina tradicional: definiciones [Internet]. Ginebra: Organización Mundial de la Salud [fecha de consulta: 15 de mayo de 2016]. Disponible en: http://www.who.int/topics/traditional_medicine/definitions/es/

9. Medicina tradicional [Internet]. Ginebra: Organización Mundial de la Salud [fecha de consulta: 15 de mayo de 2016]. Disponible en: http://www. who.int/topics/traditional_medicine/es/

10. Spadacio C, Barros de Felice N. Use of complementary and alternative medicine by cancer patients: systematic review. Rev Saude Publica. 2008;42(1):158-64.

11. Deng G, Frenkel M, Cohen L, Cassileth B, Abrams D, Capodice J, et al. Evidence-based clinical practice guidelines for integrative oncology: complementary therapies and botanicals. J Soc Integr Oncol. 2009;7(3):85-120.

12. Cirilo-Aguilar BG, Cantú-Martínez PC, Verde-Star MJ, Mata-Cárdenas B. Uso de la herbolaria en el cuidado de la salud. RESPYN Rev Salud Pública y Nutrición. 2011;12(2):Abril-Junio.

13. Lozaya X. La medicina tradicional en la realidad político-social de México [Internet]. México: Revista de Cultura Científica, Facultad de Ciencias, Universidad Nacional Autónoma de México [fecha de publicación: 1989]. Disponible en: http://www.revistaciencias.unam.mx/es/159-revistas/revista-ciencias-14/1377-la-medicina-tradicional-en-la-realidad-pol\%C3\%ADtico-social-de-m\%C3\%A9xico.html

14. Greenlee H, Balneaves LG, Carlson LE, Cohen M, Deng G, Hershman D, et al.; Society for Integrative Oncology. Clinical practice guidelines on the use of integrative therapies as supportive care in patients treated for breast cancer. J Natl Cancer Inst Monogr. 2014;50: 346-58.

15. Organización Mundial de la Salud. Estrategia de la OMS sobre medicina tradicional 2014-2023. Ginebra: Ediciones de la OMS; 2013.

16. Klafke N, Eliott JA, Wittert GA, Olver N. Prevalence and predictors of complementary and alternative medicine (CAM) use by men in Australian cancer outpatient services. Ann Oncol. 2012;23(6):1571-8. 
17. Horneber M, Bueschel G, Dennert G, Less D, Ritter E, Zwahlen M. How many cancer patients use complementary and alternative medicine: a systematic review and metaanalysis. Integr Cancer Ther. 2012;11(3): 187-203

18. Tascilar M, de Jong FA, Verweij J, Mathijssen RH. Complementary and alternative medicine during cancer treatment: beyond innocence. Oncologist. 2006;11(7):732-41.

19. Gomez-Martinez R, Tlacuilo-Parra A, Garibaldi-Covarrubias R. Use of complementary and alternative medicine in children with cancer in Occidental, Mexico. Pediatr Blood Cancer. 2007;49(6):820-3.

20. Jaime-Pérez JC, Chapa-Rodríguez A Rodríguez-Martínez M Colunga-Pedraza PR, Marfil-Rivera LJ, Gómez-Almaguer D. Use of complementary and alternative medicine by patients with hematological diseases: experience at a university hospital in northeast México. Rev Bras Hematol Hemoter. 2012;34(2):103-8.

21. Barnes PM, Bloom B, Nahin RL. Complementary and alternative medicine use among adults and children: United States, 2007. Natl Health Stat Report. 2008;10(12):1-23.

22. Molassiotis A, Fernandez-Ortega P, Pud D, Ozde G, Scott JA, Panteli V, et al. Use of complementary of alternative medicine in cancer patients: A European survey. Ann Oncol. 2005;16(4):655-63.

23. Gerson-Cwilich R, Serrano-Olvera A, Villalobos-Prieto A. Complementary and alternative medicine (CAM) in Mexican patients with cancer. Clin Transl Oncol. 2006;8(3):200-7.

24. Al-Naggar RA, Bobrychev $\mathrm{Y}$, Abdulgani M, Ramohan S, Osman MT, Abdul SY. Complementary/Alternative Medicine use among cancer patients in Malaysia. World J Med Sci. 2013;8(2):157-64.
25. McLay JS, Stewart D, George J, Rore C, Heys SD. Complementary and alternative medicines use by Scottish women with breast cancer. What, why and the potential for drug interactions? Eur J Clin Pharmacol. 2012; 68(5):811-19.

26. Kremser T, Evans A, Moores A, Luxford K, Begbie S, Bensoussan A et al. Use of complementary therapies by Australian women with breast cancer. Breast. 2008;17(4):387-94.

27. Barnes PM, Powell-Griner E, McFann K, Nahin RL. Complementary and alternative medicine use among adults: United States, 2002. Adv Data. 2004;27(343):1-19.

28. Álvarez-Hernández E, Casasola-Vargas JC, Lino-Pérez L, Burgos-Vargas R, Vázquez-Mellado J. Frecuencia de uso de medicinas complementarias y alternativas en sujetos que acuden por primera vez al servicio de reumatología. Análisis de 800 casos. Reumatol Clin. 2006;2(4):183-9.

29. Downer SM, Cody MM, McCluskey P, Wilson PD, Arnott SJ, Lister TA et al. Pursuit and practice of complementary therapies by cancer patients receiving conventional treatment. BMJ. 1994;309(6947):86-9.

30. Molassiotis A, Panteli V, Patiraki E, Ozden G, Platin N, Madsen E, et al. Complementary and alternative medicine use in lung cancer patients in eight European countries. Complement Ther Clin Pract. 2006:12(1):34-9.

31. Sánchez R, lbañez C, Suárez A. Utilización de terapias complementarias y alternativas en niños con cáncer. Rev Salud Pública. 2015; 17(5):699-712.

32. Alternative medicine spending soars [Internet]. The Washington Post [fecha de publicación: 30 de julio de 2009]. Disponible en: http://voices. washingtonpost.com/checkup/2009/07/alternative_medicine_spending. html 